\title{
CAN A TELEOPERATED ANDROID REPRESENT PERSONAL PRESENCE? - A CASE STUDY WITH CHILDREN
}

\author{
Shuichi NISHIO, Hiroshi ISHIGURO, and Norihiro HAGITA \\ ATR Intelligent Robotics and Communication Laboratories, Japan
}

\begin{abstract}
Our purpose is to investigate the key elements for representing personal presence, which is the sense of being with a certain individual. A case study is reported in which children performed daily conversational tasks with a geminoid, a teleoperated android robot that resembles a living individual. Different responses to the geminoid and the original person are examined, especially concentrating on the case where the target child was the daughter of the geminoid source. Results showed that children gradually became adapted to conversation with the geminoid, but the operator's personal presence was not completely represented. Further research topics on the adaptation process to androids and on seeking for the key elements on personal presence are discussed.
\end{abstract}

Key words: personal presence, android robot, teleoperation

What creates a person's sense of presence? When we are having a conversation or watching a movie with somebody we know, we feel that that person, not just an anonymous individual, is beside us. Fluctuating moods or emotions are factors, and personality traits are others that are consistent and forever. These factors and how they appear have been extensively studied, mainly in the field of psychology, through the analysis of various human behaviors. Is the combination of these factors powerful enough to describe and capture the individual differences in each person? Can current technology represent, record, and playback this individual sense of presence? Many studies have grappled with this question, including how well current transmission technologies, such as telephones, TV conferencing systems, or newly developed computer mediated communication (CMC) systems can approximate face-to-face communications (Wainfan, 2005). For the sense of presence, based on classical studies (Goffman, 1963), much work has pursued its nature. Co-presence, the sense of being with somebody else in a remote environment, has especially been examined in the field of virtual reality (Lombard, 1997; Zhao, 2003). These studies, however, have mainly focused on the typical, anonymous nature generally seen and not on the details specific to each individual.

Another scheme, the constructive approach, builds a hypothesis and examines the issue through implementation (Ishiguro, 2002). In the field of robotics, interest continues to grow in the social aspects of human-robot interaction. One field of keen interest is the usage of robots as a communication interface device, whose main purpose is not for

This work was supported in part by the Ministry of Internal Affairs and Communications of Japan.

Correspondence concerning this article should be addressed to Mr. Shuichi Nishio, 2-2-2 Hikaridai, Keihanna Science City, Kyoto 619-0288, Japan (e-mail: nishio@ieee.org) 
industry or as a carrier. These studies focus on robots as an informational interface with a physical presence in the real world and try to enrich their humanlike functionality, such as making gestures, eye contact, or even expressing personality and emotion. Studies have shown the importance of humanlike nonverbal channels and the superiority of the physical presence of robots to software agents or computer terminals in everyday conversation (Fong, Nourbakhsh, \& Dautenhahn, 2003; Kanda, Ishiguro, Imai, \& Ono, 2004). Appearance remains the one difference between human beings. Recent manufacturing advances have produced android robots whose appearance is quite similar to humans, and several studies have begun (Ishiguro, 2005).

Based on these studies, we seek to clarify the key elements required in representing and perceiving the sense of presence that each individual holds: personal presence. We believe that such findings will lead to a deeper understanding of human nature, and at the same time, provide a means to build a robot that can communicate more effectively with human beings.

However, with traditional robots, one serious issue existed when using them as a means to study human natures: intelligence. Although actively studied, it is impossible with current technology to build a robot that behaves and talks like human beings. This issue prevents researchers from conducting effective examinations on the characteristics of human nature that can only be seen through intelligent conversations. To overcome this 'intelligence' issue, we have developed a new android system called geminoid, which is a teleoperated robotic system with an android robot that looks and behaves similar to a person.

As a first step in this study toward the inspection of the nature of personal presence, we conducted a case study using the class of participants most sensitive to personal presence: members of one's family. And since children, rather than adults, can show us more direct responses, we conducted this case study with two participants who possess a special relation to the geminoid: the daughter of the model person and a 4-year-old boy who didn't know the model person. Previous studies with the android robots mainly focused on the very first impression on meeting androids (Minato, Shimada, Ishiguro, \& Itakura, 2004; MacDorman \& Ishiguro, 2006). In this study, we focus on seeking two issues: 1) to see how the participant gets adapted to the geminoid, or how the attitudes changed through daily conversation experiences, and 2) how well one's personal presence can be represented through the geminoid system. These also include seeking the elements that can effectively measure how well personal presence is represented.

\section{THE GEMINOID SySTEM}

Here we briefly describe an overview of the geminoid system (Nishio, Ishiguro, \& Hagita, 2007). A geminoid is a robot that functions as a duplicate of a living person. It appears and behaves like that person and is connected to the person by a computer network. Geminoids extend the applicable field of android science. Androids are designed for studying human nature in general. With geminoids, we can study such 

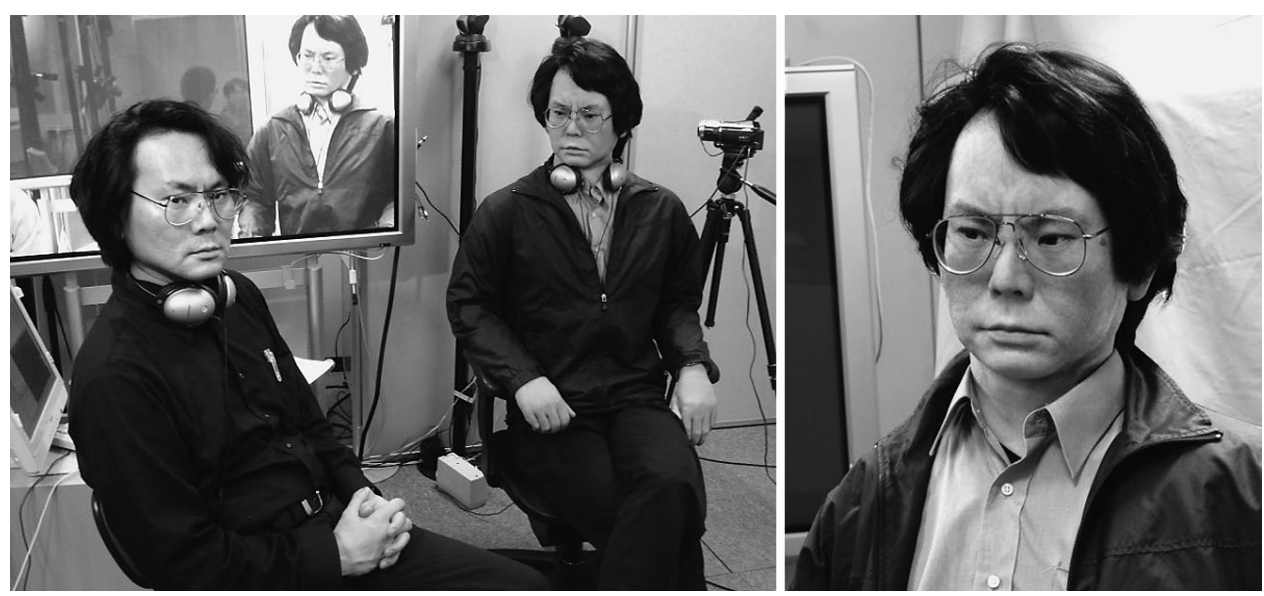

Fig. 1. Geminoid HI-1 and its original person.

personal aspects as presence or personality traits, tracing their origins and implementation into robots. Figure 1 shows the robotic part of HI-1, the first geminoid prototype. The geminoid's appearance is based on a living person and does not depend on the imagination of designers. Its movements can be made or evaluated simply by referring to the original person. The existence of a real person analogous to the robot simplifies comparison studies.

The robotic element has essentially identical structure as previous androids (Ishiguro, 2005). However, efforts concentrated on making a robot that appears - not just to resemble a living person - to be a copy of the original person. Silicone skin was molded by a cast taken from the original person; shape adjustments and skin textures were painted manually based on MRI scans and photographs. Fifty pneumatic actuators drive the robot to generate smooth and quiet movements, which are important attributes when interacting with humans. The allocations of actuators were determined so that the resulting robot can effectively show the necessary movements for human interaction and simultaneously express the original person's personality traits. Among the 50 actuators, 13 are embedded in the face, 15 in the torso, and the remaining 22 move the arms and legs.

Since geminoids are equipped with teleoperation functionality, they are driven by more than an autonomous program. By introducing manual control, the limitations in current AI technologies can be avoided, enabling long-term, conversational human-robot interaction experiments. Figure 2 shows the teleoperation interface. Two monitors show the controlled robot and its surroundings, and microphones and a headphone are used to capture and transmit utterances. The captured sounds are encoded and transmitted to the geminoid server by IP links from the interface to the robot and vice versa. The operator's lip corner positions are measured by an infrared motion capturing system in real time, converted to motion commands, and sent to the geminoid server by the network. This enables the operator to implicitly generate suitable lip movement on the robot while speaking. 


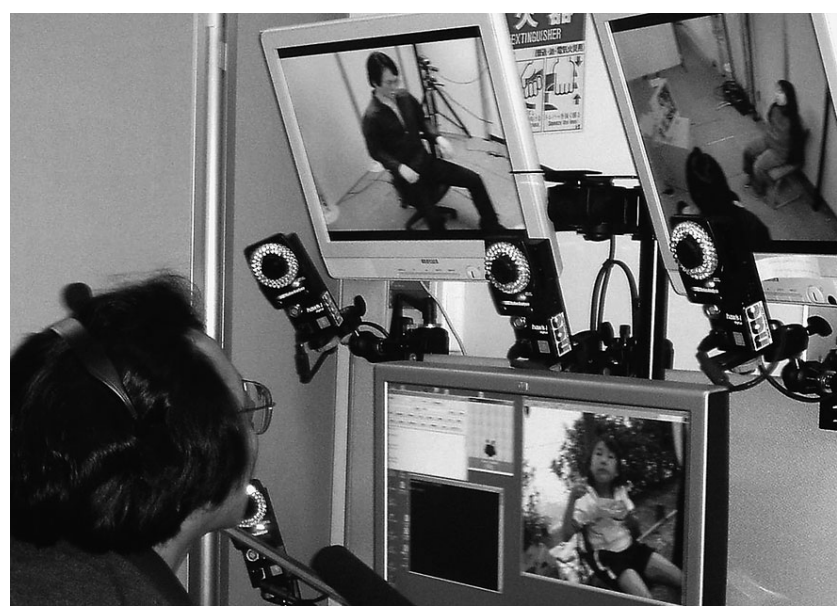

Fig. 2. Geminoid teleoperation console.

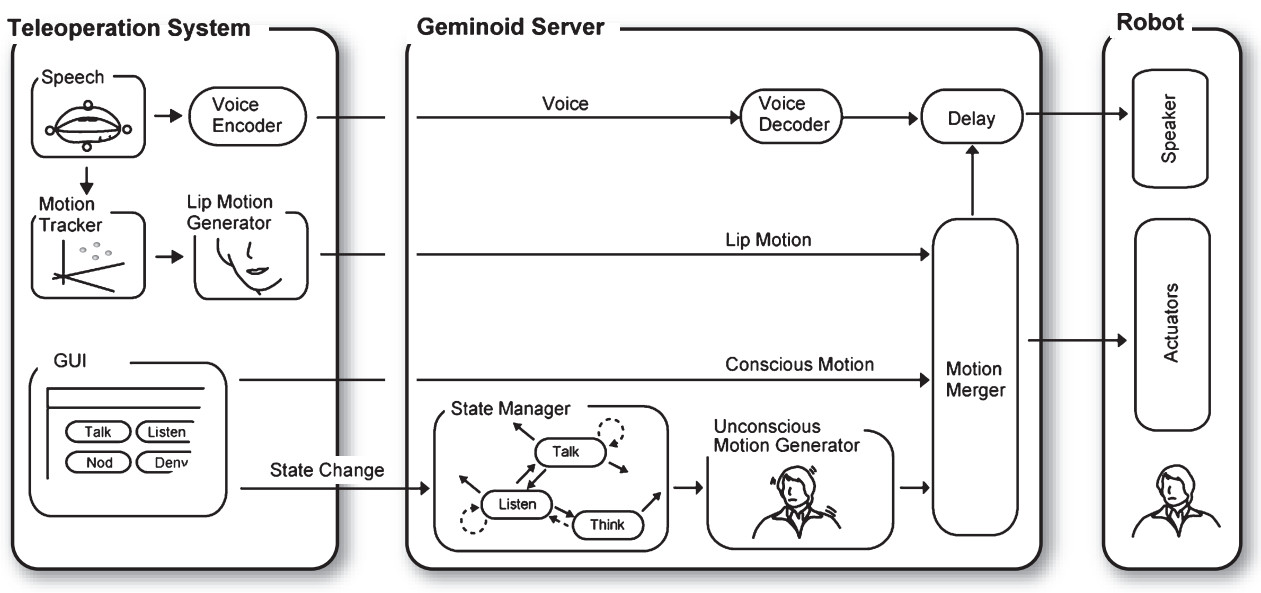

Fig. 3. Block diagram of the geminoid system.

The geminoid server receives robot control commands and sound data from the teleoperation interface, adjusts and merges inputs, and sends and receives primitive controlling commands between the robot hardware. Figure 3 shows the major data flow in the geminoid system. As the robot's features become more humanlike, its behavior should also become suitably sophisticated to retain a natural look (Minato et al., 2006). One thing that can be seen in every human being, and that most robots lack, are the slight body movements caused by its autonomous system, such as breathing or blinking. To increase the android's naturalness, the geminoid server emulates the human autonomous system and automatically generates these micro-movements. Such automatic robot motions are merged with explicit operation commands sent from the remote console. 


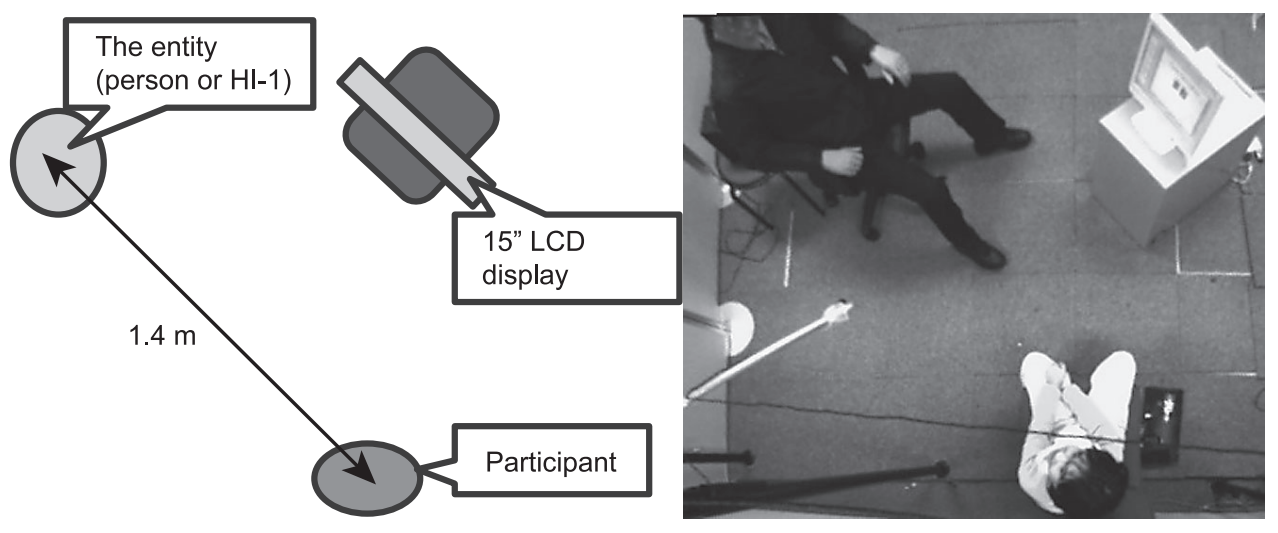

Fig. 4. Experiment room setup.

\section{METHODS}

\section{Participants}

Two children joined the experiment, a girl (R) and a boy (K). Neither had never seen or heard about the geminoid before the experiment, and they were not told that the features of geminoid HI-1 were based on Dr. Ishiguro or that he was teleoperating it.

$\mathrm{R}$ is a 10-year-old, elementary school student. She is also the daughter of the geminoid model person. In the past, she modeled for a child android, Repliee Rl (Minato, et al., 2004), and had joined some humanoid robot experiments. Her parents describe her as shy. $\mathrm{K}$ is a 4-year-old boy and the son of one of the authors. $\mathrm{He}$ is outgoing and rarely becomes anxious even when meeting somebody for the first time. On several occasions, $\mathrm{K}$ has seen and played with humanoid robots at exhibitions, but has never seen androids of any kind before this experiment.

\section{Procedures}

After participants were led to the experimental room by the experimenter, they engaged in conversational tasks with the other entity in the room. The participants were seated in front of a 15" LCD display. The distance between the participants and the entity was approximately $1.4 \mathrm{~m}$. Figure 4 shows the seat alignment.

Two conditions were compared. In the first case, the entity was a person, the original of the geminoid HI-1, Dr. Ishiguro ( $P$ condition). In the latter case, the participants had conversations with geminoid HI-1, which was remotely operated by the same person as in the $\mathrm{P}$ condition ( $G$ condition). In the $\mathrm{P}$ condition, the entity was told to limit his movements, so that his motion would resemble HI-1 in the G condition. Each condition was conducted twice for each participant. Thus, eight sessions were held.

Several conversational tasks were chosen, based on their parents' opinions and from pretest observations. These tasks reflected the children's interests and ages, so they could participate without becoming bored during the sessions. For R especially, some chosen tasks required conversation about family memories. Thus, a different set of tasks was chosen for each participant. R's tasks were the following:

1) Photo: Viewing a series of family photographs and talking about them.

2) Shiri-tori: Playing a Japanese word game in which players are required to say a word that begins with the last letter of the previous word.

3) Video: Watching video in which $\mathrm{R}$ or her father appears and talking about them. Clips from TV programs were used.

4) Math: Doing simple math problems. The entity gave questions and R answered.

$\mathrm{K}$ 's tasks were the following: 


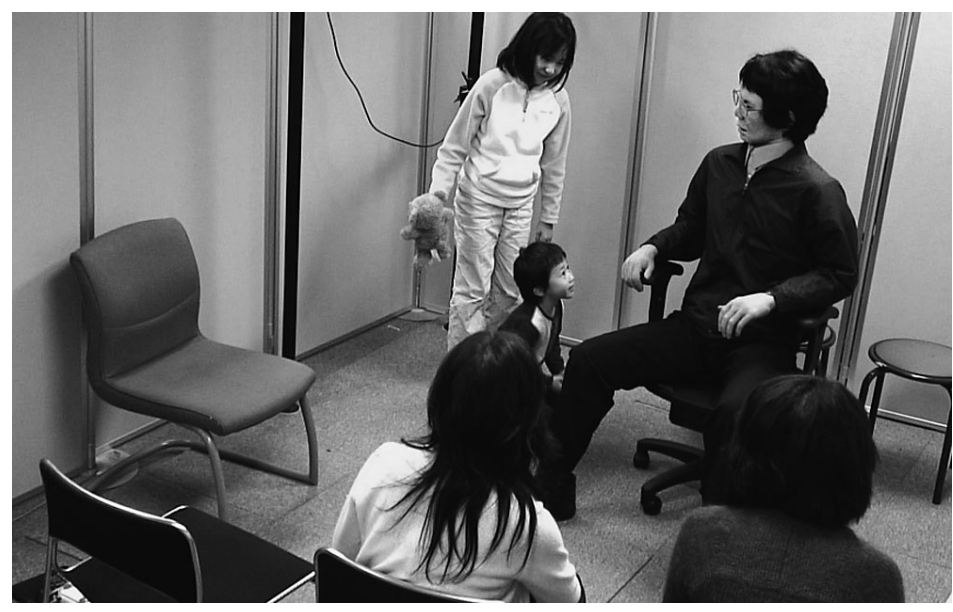

Fig. 5. Habituation session on the second day.

1) Video: Watching family videos and talking about them.

2) Talk: Talking about some recent issues related to the participant. In the experiment, several topics were chosen by K's father (one of the experimenters).

3) Movie: Watching movie clips and talking about them.

4) English: Counting or reciting the alphabet in English.

After these tasks, several additional tasks were performed as a trial for future experiments. Since these tasks were only conducted as a trial for future experiments, they were not analyzed in this paper.

Since each task lasted until the entity decided that the participant was getting bored with the task, the duration of each task was not strictly controlled. The average duration time of each task was approximately three minutes, and the average session time, including the unanalyzed tasks, was approximately 20 minutes.

At the beginning of each task, the entity asked the LCD display for the next task, and then the name of each task was shown on the display. For the 'talk' task, a conversation topic was also displayed. During the 'photo', 'video,' and 'movie' tasks, images or movies were shown on the display. For example, in the 'photo' task, several photographs were shown. Changing the displayed photograph was done by the experimenter in a separate room. In the $\mathrm{G}$ condition, the same contents were also shown on the teleoperation console (Fig. 2).

After each session, participants were interviewed about their impressions of the test and the entity they were talking with. To relax the children, they were interviewed by one of their parents. At the end of each experiment day, several additional questions inquired about comparisons between the geminoid and the real person.

The main experiment was held over two days. On the first day, four sessions were conducted in the following order: R-P (participant R in the P condition), K-P, R-G, and K-G. Two weeks later, on the second day, another four sessions were conducted in the order of K-G, R-G, R-P, and K-P. One week before the first day, test sessions were conducted with identical participants, but only for the $\mathrm{P}$ condition to determine the effectiveness of the tasks and help the participants become habituated to the experimental environment. To help them relax, at the beginning of the first day, the children spent some time in the experiment room with their mothers reading or playing. During this first habituation period, the android was hidden. Also, at the beginning of the second day, the participants and their mothers spent some time talking and playing with the HI-1 (Fig. 5).

\section{Measures}

Although various studies have been conducted for seeking an measure on the effect of CMC (Wainfan, 2005) or presence (Prothero, Parker, Furness, \& Wells, 1995), no effective measure is yet known for personal presence. Thus, we tried some measures for capturing the participants' impression toward the entity in 
conversation. Each experimental session was recorded by video cameras to measure the following data:

1) Eloquence of Conversation

The amount of conversational utterances is known to be influenced by the participant's emotional state and impression to whom s/he is having conversation with (Leary 1983, Nishida, Ura, Kuwabara, \& Kayanno, 1988). The conversations in each session were transcribed from the audio recordings. Since all conversations were done in Japanese, we analyzed the transcripts to see how actively participants spoke in each task, which is similar to counting words in English sentences. Here, the transcripts were morphologically analyzed and split into tokens by the Chasen tokenizer (Asahara \& Matsumoto, 2000). The numbers of extracted tokens were counted for each participant or entity for each task. The following relative eloquence rate was derived to see how actively each participant spoke:

$$
\gamma_{\text {eloquence }}=\frac{\text { total number of tokens in participant speech }}{\text { total number of tokens in entity speech }}
$$

\section{2) Gaze direction}

Nonverbal behaviors, such as inter-personal distance, gestures, eye contact rate or body movements are another factor that are influenced by the participant's impression to whom s/he is talking with (Feyereisen 1982, Planalp 1999). Thus, we here chose two measures, eye contact rate and body movement. From image recordings, the gaze directions of both the entities and the participants were observed and coded into two categories: watching another (eye contact) or not. From these data, we derived the relative eye contact rate, defined as follows:

$$
\gamma_{\text {ey contact }}=\frac{\text { total duration of eye contact }}{\text { total duration of the entity watching the participant }}
$$

\section{3) Body movement}

As a simple measure to evaluate how the attitude of participants changed, we calculated the body movement amount of each participant from video images. This is to roughly see the changes in participants' nonverbal behaviors such as inter-personal distance or the amount of gestures. We took the sum of the motion vector norm obtained by performing a block matching calculation between subsequent frames for the image region in which the participant appeared. The block size was set to $8 \times 8\left(\right.$ pixel $\left.^{2}\right)$. Due to restrictions in the experimental setting, camera angles differed between sessions. Also, the body size of the two participants differed. Since the sum of the motion vector norms depends on the body area size, the obtained values were normalized by the standard area of each participant. This area was obtained from a video frame showing each participant in neutral poses. The motion amount was first calculated frame-by-frame (29.97 frames/sec) and then totaled in one-second intervals.

\section{RESULTS}

As stated before, this experiment is a case study with only two subjects and a limited number of trials. Thus, we did not conduct any statistical analysis of the measured values. Instead, in this section we describe the subjective tendencies observed from the results.

\section{Eloquence of Conversation}

Figure 6 shows the relative eloquence measures extracted from each task. In the 'English' task, K was asked to count or recite the alphabet in English. Thus, the amount of speech does not show meaningful values, and so the values of the 'English' task are omitted.

For both participants, the results in the $\mathrm{G}$ conditions seem to be lower than the $\mathrm{P}$ 


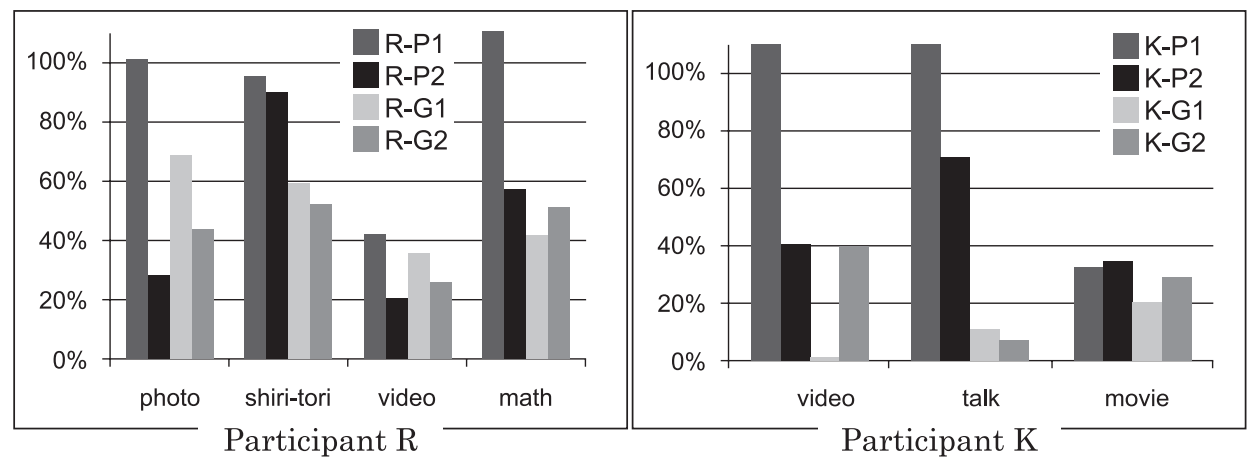

Fig. 6. Relative eloquence rate.

conditions. For R, the differences between each condition are rather weak. Relative eloquence in the $\mathrm{G}$ conditions seems to be significantly lower than in the P condition only in the 'shiri-tori' task (R).

As for $\mathrm{K}$, the overall rate is clearly lower in the G conditions. Especially in the first 'video' task of session G-1, where the participant met the geminoid for the first time, the rate was around $1 \%$, which indicates that the participant remained mostly quiet throughout the task. The high value of over $100 \%$ in the same task at session P-1 shows a high contrast between the two conditions. We can see, however, in G-2, a tendency where the value is recovering, and the differences between P-2 and G-2 have almost vanished. In the 'movie' task for $\mathrm{K}$, the differences among conditions are much smaller, perhaps reflecting the nature of the task. It seems that the participant's attention was focused on the movie, and his overall response was low, as can also be seen in other measures.

\section{Eye Contact}

The results are shown in Figure 7. For R, the values of the $\mathrm{G}$ conditions seem to be lower, except in the 'photo' task. As for K, no clear difference between the two conditions can be found.

\section{Body Movement}

Figure 8 shows the average body movements. The detailed temporal changes in the first tasks for each participant are shown in Figure 9. For R, the overall amount is smaller in P-1 and G-1, compared to P-2 and G-2. The values of participant $\mathrm{K}$ seem to show a clearer tendency. Obviously, the values in the G-1 session are much smaller compared to the other sessions, except in the 'English' and 'movie' tasks. This tendency matches impressions from the recorded video images. In the G-1 session, $\mathrm{K}$ stayed still throughout the task. In contrast, in the $\mathrm{P}$ condition tasks, $\mathrm{K}$ always kept moving, spoke a lot, and showed rich facial expressions. The only exception is when he was watching movies; he concentrated on the movie and remained still in his seat.

In the G-1 session, K's body motion average is large in the 'English' task. Watching the video, we found that $\mathrm{K}$ began to make large movements, frequently looking at the 

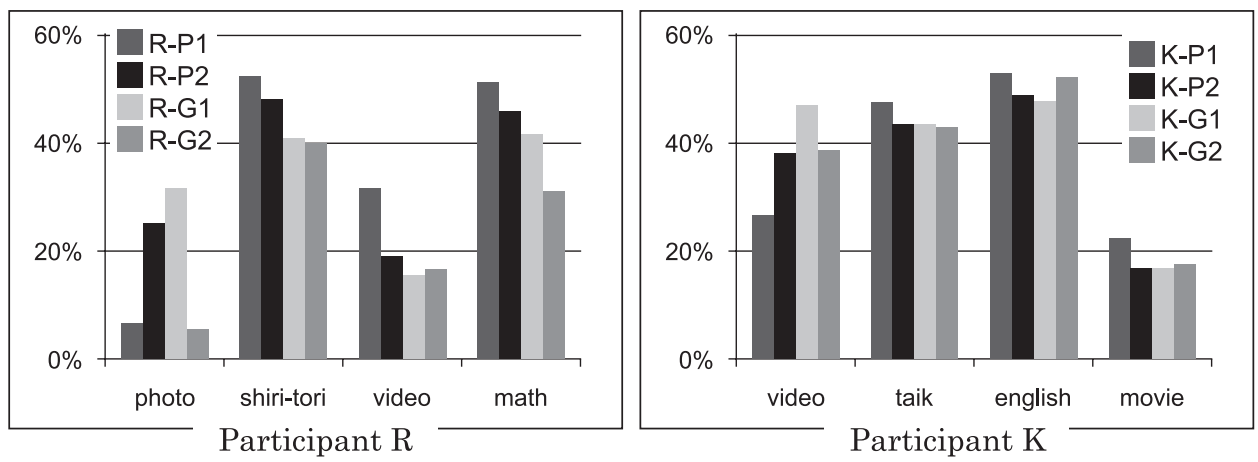

Fig. 7. Relative eye contact rate.

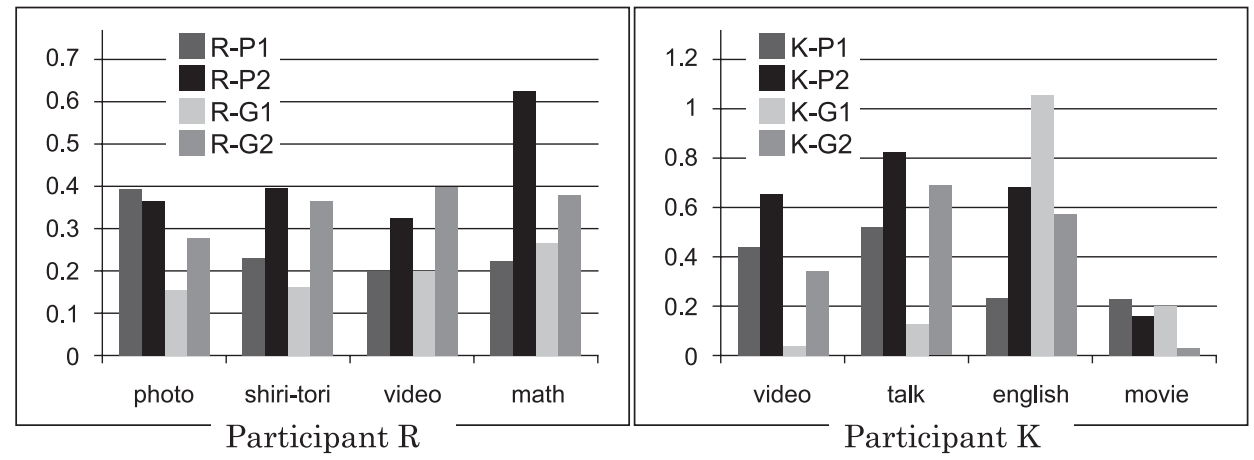

Fig. 8. Average body motion

room exit. He might have become tired from the anxiety of the previous two tasks. In the G-2 session, the values seem to recover to the values of the P conditions.

\section{Interviews}

Even though both participants were scared by the geminoid, they turned out to have quite different impressions. The answers in the first and second days were almost identical for both participants.

$\mathrm{R}$ seemed to notice that the geminoid was a robot controlled by her father in a different place. Most of her impressions were based on this finding. She described the geminoid as scary, mainly because its features and movements were strange. She said that the geminoid did not look like her farther, but she could not specify which part was strange or different. She preferred her real father to his geminoid because "this robot can't play Wee (the name of a portable game player) with me, and it can't grab things."

$\mathrm{K}$ had a different impression. After the first $\mathrm{P}$ session, $\mathrm{K}$ insisted that the entity (the real Dr. Ishiguro) was a robot. He thought that it was alive and breathing, and its (his) figure was normal, but he still felt that it was a robot. He described the "robot" as very 

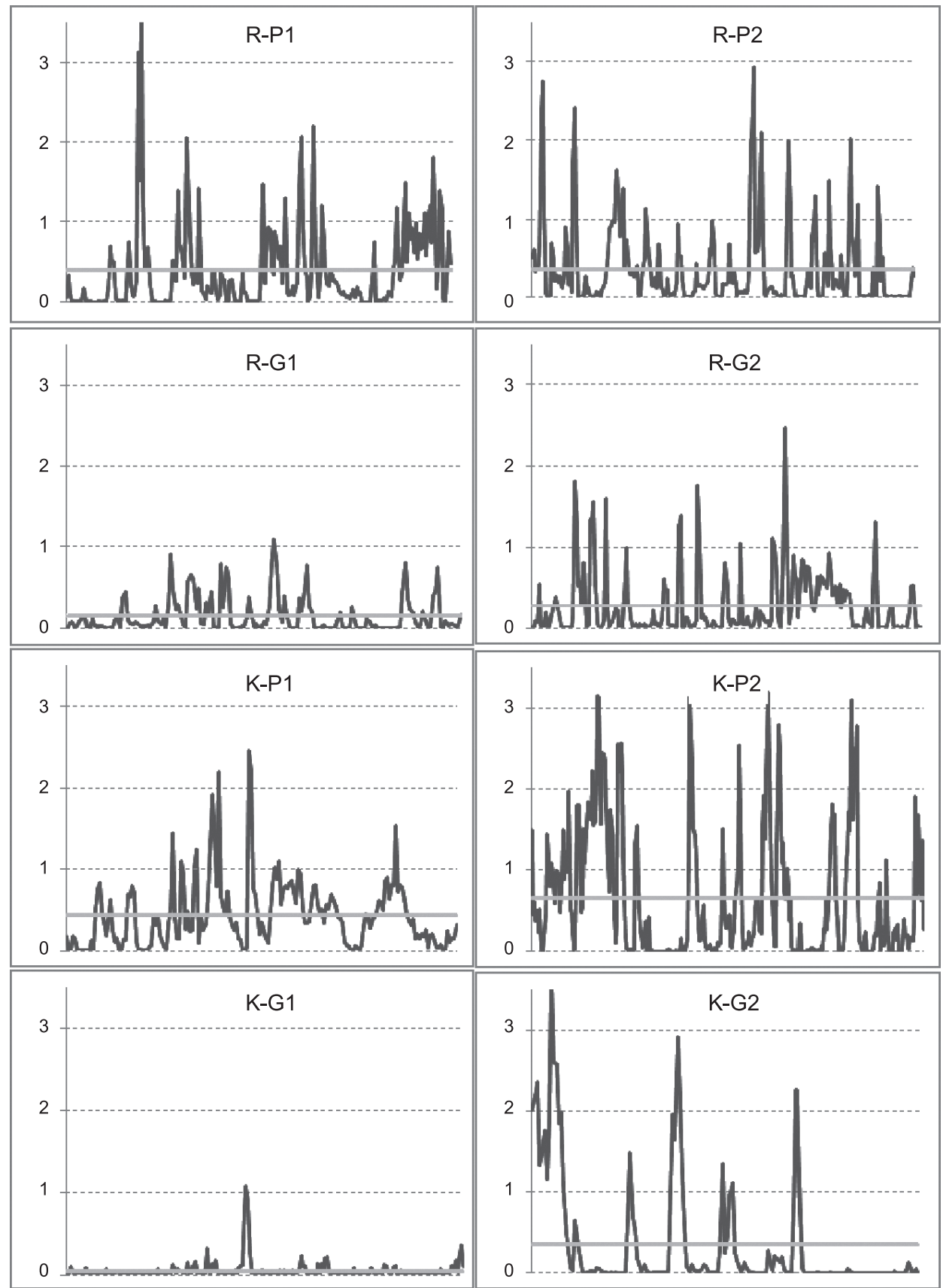

Fig. 9. Body movement changes. Here the temporal changes while the first task in each session is shown ('photo' task for R and 'video' task for K). The horizontal line indicates the average level. 
"serious" and said that the entity listened well to his story and that he would like to play with 'it'. But after the first G session, K said, "I thought the first one was a robot, but that was a mistake. This one must be a robot." $\mathrm{K}$ believed that the entity (HI-1) kept wearing a mask and said "it was very scary, because it had a very thin nose." He also mentioned that it was not breathing, and its mouth was not moving well when it was speaking.

$\mathrm{K}$ seemed to be rather confused by his experience. He sometimes mentioned that the "man" (HI-1) was a robot, but later he thought it was "a person" wearing a strange mask. "He should take off his strange mask that he keeps wearing," K said.

\section{Discussion}

\section{Adaptation to the Geminoid}

Although the participants described the geminoid as scary identically in both trials, the measurement results seem to show that the participants were gradually adapted to conversation through the geminoid. This was especially clear from the video recording of $\mathrm{K}$, where he remained silent and still in the first half of the first $\mathrm{G}$ condition, and then gradually became active, as his normal manner. The fact that the younger participant of 4 years old was much scared with the geminoid seems to follow the result in the previous study (Minato et al., 2004). The results in this study also showed that, even the younger aged, sensitive participant would be adapted to the uncanny appearance of the geminoid through the conversation with the entity. Although the responses in both participants toward the geminoid became close to the response toward the real person, there still remained some differences, especially in the younger participant $\mathrm{K}$. One influencing factor might be the expressiveness in facial expressions. As seen in K's comment where he thought the geminoid was wearing a mask, the facial expressiveness of the android body must be improved. Even if efforts are made to create a replica of an existing person, both in their appearance and behavior, many differences still exist in the geminoid that a human being will not show. However, even though issues remain for the geminoid function before it attains the complex functionality of human beings, we can see from the measured data that both children gradually adapted to conversation with the geminoid. Recall that even with real human beings we sometime experience an uncanny feeling that decreases or disappears as we spend more time with that person. Similar to the developmental process in infants, where perceptual functions become optimized to frequent stimuli (Kuhl, Tsao, \& Liu, 2003; Pascalis, Haan, \& Nelson, 2002), the classification function within ourselves might get 'personalized' or tuned to the behavior or expression that each specific person shows.

Has the same adaptation process worked through the conversations with the geminoid? Further examination on this adaptation process will lead to understanding the nature of human-robot interaction, and also to new findings in human developmental process. 


\section{Representing Personal Presence}

As for the operator's personal presence, it was not fully represented at identical levels as the real person. In the case of the daughter, the voice and the content of the speech seems to lead to a quick concluding that the geminoid was operated by her father. This is the same phenomena seen in many adult visitors to our laboratory. When they first see the geminoid, they get surprised and have some uncanny feelings. But after having conversation for a while, they get used to it, and feel just like talking with the real person (Nishio et al., 2007). However, even she came to know that the geminoid was operated by her father, the measurements still showed slight differences between talking with the real father and the operated geminoid.

This was much clearly seen for K, the participant unfamiliar to the geminoid source. Here even presenting the identification of the operator seemed to fail. In the interview after the first day, K said that the geminoid was definitely somebody that he had never met before, although he had no problem in recognizing the real entity as the same person in the two experiment days.

From the measurement values that $\mathrm{K}$ had shown, we can think that $\mathrm{K}$ was also gradually adapted to the geminoid, and his attitudes were getting closer to that of the real person. But we can also think that, the behavior and appearance of the geminoid was showing some different sense of personal presence to $\mathrm{K}$. In case of $\mathrm{R}$, who knew the source person quite well, her belief that the geminoid was operated by her father might overcame her impression on the geminoid. But for $\mathrm{K}$, who was not familiar with the operator, what he saw for each entity might lead to a stronger impression than the content of their speech. The results with the two participants, R and K, seem to show that different aspects of the geminoid were focused by each of the participants, in forming their impression on the presence of the geminoid.

The results in this study showed that the geminoid is still not perfect in representing personal presence. Although participants got gradually adapted to having conversation with the geminoid, the impression they felt from the geminoid was not the same as its original source. What are them that define an individual? And what further do we need to represent the presence of an individual? There are many elements that are believed to show individuality. From appearance, voice, ways of speaking, or even gating pattern, we believe that we can identify a person. In the current geminoid, some elements, such as its voice, speech content or memories, are identical with the original person as the teleoperation system is used. Some are quite close to the source, such as its appearance, and some, such as facial expression, are still not close enough, mainly due to engineering issues. Naively, it seems to be easy to express individuality when the appearance of the entity is close to the original and the speech content is exactly that of the original. But the results in this study showed that these were not enough. The fact that no difference was seen in the eye contact rate seems to show that the geminoid is in one aspect superior to telephone or CMC systems (Wainfan, 2005). But in total, the current geminoid is not as good as other systems for correctly transmitting individuality. We need to seek further, for measurements and elements where personal presence can be described and defined. By utilizing the function of the geminoid system, where various elements that possibly form 
the personal presence of an individual can be added or subtracted, further study will lead us to build robots to better represent humanlike presence, and also to clarify the key elements that makes a person to be an individual being.

\section{REFERENCES}

Asahara, M., \& Matsumoto, Y. 2000. Extended models and tools for high-performance part-of-speech tagger. In Proceedings of International Committee on Computational Linguistics (COLING), 21-27.

Feyereisen, P. 1982. Temporal distribution of co-verbal hand movements. Ethology and Sociobiology, 3, $1-$ 9.

Fong, T., Nourbakhsh, I., \& Dautenhahn, K. 2003. A survey of socially interactive robots. Robotics and Autonomous Systems, 42, 143-166.

Goffman, E. 1963. Behavior in public places. New York: The Free Press.

Ishiguro, H. 2002. Toward interactive humanoid robots: a constructive approach to developing intelligent robot, In Proc. $1^{\text {st }}$ International Joint Conference on Autonomous Agents and Multiagent Systems, $621-622$.

Ishiguro, H. 2005. Android Science: Toward a new cross-disciplinary framework. In Proceedings of Toward Social Mechanisms of Android Science: A CogSci 2005 Workshop, 1-6.

Kanda, T., Ishiguro, H., Imai, M., \& Ono, T. 2004. Development and evaluation of interactive humanoid robots. Proceedings of the IEEE, 92, 1839-1850.

Kuhl, P. K., Tsao. F. M., \& Liu, H. M. 2003. Foreign-language experience in infancy: Effects of short-term exposure and social interaction on phonetic learning. Proceedings of the National Academy of Sciences, 100, 9096-9101.

Leary, M. R. 1983. Understanding social anxiety. Beverly HiUs: Sage.

Lombard, M. 1997. At the heart of it all: The concept of presence. Journal of Computer-Mediated Communication, 3, 000-000.

MacDorman, K. F., \& Ishiguro, H. 2006. The uncanny advantage of using androids in social and cognitive science research. Interaction Studies, 7, 297-337.

Minato, T., Shimada, M., Ishiguro, H., \& Itakura, S. 2004. Development of an Android Robot for Studying Human-Robot Interaction. In Proceedings of the Seventeenth International Conference on Industrial and Engineering Applications of Artificial Intelligence and Expert Systems (IEA/AIE), 424-434.

Nishida, K., Ura, M., Kuwabara, T., \& Kayanno, J. 1988. Intermediating influence of conversation on social interaction. Research in Social Psychology, 3, 46-55.

Nishio, S., Ishiguro, H., \& Hagita, N. 2007. Geminoid: Teleoperated android of an existing person. In de Pina Filho, A. C. (Ed)., Humanoid Robots. Vienna: I-Tech Education and Publishing.

Pascalis, O., Haan, M., \& Nelson, C. A. 2002. Is Face Processing Species-Specific During the First Year of Life? Science, 296, 1321-1323.

Planalp, S. 1999. Communicating emotion: social, moral, and cultural processes. New York: Cambridge University Press.

Prothero, J., Parker, D., Furness, T., \& Wells, M. 1995. Towards a robust, quantitative measure for presence. In Proceedings of the Conference on Experimental Analysis and Measurement of Situation Awareness, 359-366.

Wainfan, L. 2005. Challenges in virtual collaboration: Videoconferencing, audioconferencing and computermediated communications. Santa Monica, CA: RAND Coropration.

Zhao, S. 2003. Toward a taxonomy of copresence, Presence: Teleoperators and Virtual Environments, 12, 445-455. 